\title{
A Verification Scenario of Knock-on Tail Formation due to Nuclear Plus Interference Scattering in ${ }^{3} \mathrm{He}$-Containing Deuterium Plasmas*) $^{*}$
}

\author{
Hideaki MATSUURA, Kei IWAMURA and Yasuyuki NAKAO \\ Department of Applied Quantum Physics and Nuclear Engineering, \\ Kyushu University, 744 Motooka, Fukuoka 819-0395, Japan
}

(Received 20 November 2012 / Accepted 4 April 2013)

\begin{abstract}
A scenario to verify the knock-on tail formation in deuteron distribution function by nuclear plus interference (NI) scattering and validate the Boltzmann-Fokker-Planck (BFP) model is proposed. Deuterium plasma with ${ }^{3} \mathrm{He}-$ beam injection is considered and on the basis of the BFP model, knock-on tail formation in deuteron distribution function by energetic protons and the resulting modification of the neutron emission spectrum are examined. A recognizable change in the energetic-neutron emission rate due to the knock-on tail formation is presented. The noise neutrons for the measurement arising from the $\mathrm{T}(\mathrm{d}, \mathrm{n})^{4} \mathrm{He}$ reaction is also evaluated by continuous-energy Monte Carlo transport simulation. The influence of the noise (slowing-down) neutrons on the measurement is discussed.

(c) 2013 The Japan Society of Plasma Science and Nuclear Fusion Research
\end{abstract}

Keywords: nuclear plus interference scattering, fast-ion velocity distribution function, neutron emission spectrum, ${ }^{3}$ He-beam injection, Boltzmann-Fokker-Planck equation

DOI: $10.1585 /$ pfr.8.2403064

\section{Introduction}

It is well known that in the thermonuclear plasmas the energetic ions create the knock-on tails [1-5] in fuel-ion velocity distribution functions due to nuclear plus interference (NI) scattering ${ }^{\dagger}[6,7]$. So far several calculations have predicted that in $\mathrm{D}^{3} \mathrm{He}$ plasmas the transferred power from energetic protons to bulk ions is enhanced almost three times due to the NI scattering $[8,9]$. In such a case the confinement parameter can be reduced significantly [10]. Recently we have also shown that the magnitude of the energetic component in proton slowing-down distribution in $\mathrm{D}^{3} \mathrm{He}$ plasmas is considerably overestimated if the NI scattering was ignored [11]. The contributions of the NI scattering on plasma burning can determine the essential physics of thermonuclear fusion especially in the $D^{3} \mathrm{He}$ plasmas, and it is important to experimentally ascertain the phenomena and validate the analysis model.

So far use of energetic ions for plasma diagnostic has been studied [12-14]. Ryutov [12] has pointed out that the NI scattering of alpha-particle significantly affects the distribution function of impurity ions and has suggested that the phenomenon can be utilized for diagnostics in thermonuclear plasmas. The hot ion populations created by alpha-particles or RF-heated minority ions were evaluated

author'se-mail: mat@nucl.kyushu-u.ac.jp

*) This article is based on the presentation at the 22nd International Toki Conference (ITC22).

$\dagger$ The NI scattering is defined by subtracting Coulomb contributions from experimental data. It is also referred to as "nuclear elastic scattering (NES)". in DT plasmas [13] and a scenario to measure the fast confined alpha-particle distribution using hot ions produced by NI scattering was also proposed [14]. The measurement using related techniques is planned to be implemented in ITER [15].

In deuterium plasmas, proton is intrinsically produced by the $\mathrm{D}(\mathrm{d}, \mathrm{p}) \mathrm{T}$ and subsidiary ${ }^{3} \mathrm{He}(\mathrm{d}, \mathrm{p})^{4} \mathrm{He}$ reactions. If a small amount of ${ }^{3} \mathrm{He}$ is included, for example in the minority heating experiments, the proton production rate by the ${ }^{3} \mathrm{He}(\mathrm{d}, \mathrm{p}){ }^{4} \mathrm{He}$ reaction is considerably enhanced. The fusion-produced energetic protons would contribute to the knock-on tail formation and the spectrum modification processes $[3,16,17]$. Previously we have proposed a scenario to examine the NI-scattering effects which is expected to appear in the $\mathrm{D}^{3} \mathrm{He}$ plasmas by using existing deuterium plasmas [18]. In the previous paper we tried to detect (a) non-Gaussian component of the neutron emission spectrum produced by $\mathrm{D}(\mathrm{d}, \mathrm{n})^{3} \mathrm{He}$ reactions and/or $(\mathrm{b})$ $0.478-\mathrm{MeV} \gamma$-ray generated by ${ }^{6} \mathrm{Li}+\mathrm{D}$ reactions in ${ }^{3} \mathrm{He}-$ conatining deuterium plasmas. In the neutron spectrum measurement scenario, the non-Gaussian component was estimated almost six orders smaller compared with the $2.45 \mathrm{MeV}$ peak. In this paper we improve the measurement scenario by adopting energetic ${ }^{3} \mathrm{He}$ beam injection and try to increase the non-Gaussian component in the neutron emission spectrum produced by $\mathrm{D}(\mathrm{d}, \mathrm{n})^{3} \mathrm{He}$ reactions.

In a deuterium plasma the $\mathrm{T}(\mathrm{d}, \mathrm{n})^{4} \mathrm{He}$ reactions are induced in parallel with the main $\mathrm{D}(\mathrm{d}, \mathrm{n})^{3} \mathrm{He}$ and $\mathrm{D}(\mathrm{d}, \mathrm{p}) \mathrm{T}$ reactions and neutrons with $\sim 14 \mathrm{MeV}$ birth energy are pro- 
duced. The slowing-down component of the neutrons produced by the $\mathrm{T}(\mathrm{d}, \mathrm{n})^{4} \mathrm{He}$ reactions may disturb the neutron spectrum formed by the $\mathrm{D}(\mathrm{d}, \mathrm{n})^{3} \mathrm{He}$ reactions. In this paper we evaluate the noise neutrons for the measurement by means of the Monte Carlo transport simulation method assuming a typical torus fusion experimental device.

We improve the previous scenario [18] and newly propose a possible experiment to validate the NI-scattering effects in $\mathrm{D}^{3} \mathrm{He}$ plasmas.

\section{Analysis Model}

\subsection{Evaluation of ion distribution functions}

The BFP equation for ion species $i$ ( $i=\mathrm{D}$, T, alphaparticle, proton and ${ }^{3} \mathrm{He}$ ) is written as

$$
\begin{aligned}
& \sum_{j}\left(\frac{\partial f_{i}}{\partial t}\right)_{j}^{\text {Coulomb }}+\sum_{n}\left(\frac{\partial f_{i}}{\partial t}\right)_{n}^{\mathrm{NI}}+\frac{1}{v^{2}} \frac{\partial}{\partial v}\left(\frac{v^{3} f_{i}}{2 \tau_{\mathrm{c}}^{*}(v)}\right) \\
& +S_{i}(v)-L_{i}(v)=0,
\end{aligned}
$$

where $f_{i}(v)$ is the velocity distribution function of the species $i$. The first term on the left-hand side of Eq. (1) represents the Coulomb collision term. The summation is taken over all background species, i.e. $j=\mathrm{D}, \mathrm{T}$, alphaparticle, proton, ${ }^{3} \mathrm{He}$ and electron. The collision term is hence non-linear, retaining collisions between ions of the same species. The electrons are assumed to be Maxwellian with temperature $T_{\mathrm{e}}$. The second term on the left-hand side of Eq. (1) accounts for the NI scattering [6] of species $i$ by background ion $n[5,17]$. We consider the NI scattering between 1) alpha-particle and $\mathrm{D}, 2$ ) alpha and $\mathrm{T}, 3$ ) alpha and $\left.{ }^{3} \mathrm{He}, 4\right)$ proton and $\left.\mathrm{D}, 5\right)$ proton and $\mathrm{T}$ and 6$)$ proton and ${ }^{3} \mathrm{He}$, i.e. $(i, n)=(\mathrm{D}, \alpha),(\mathrm{T}, \alpha),\left({ }^{3} \mathrm{He}, \alpha\right),(\alpha, \mathrm{D}),(\alpha, \mathrm{T})$, $\left(\alpha,{ }^{3} \mathrm{He}\right),(\mathrm{D}, \mathrm{p}),(\mathrm{T}, \mathrm{p}),\left({ }^{3} \mathrm{He}, \mathrm{p}\right),(\mathrm{p}, \mathrm{D}),(\mathrm{p}, \mathrm{T})$ and $\left(\mathrm{p},{ }^{3} \mathrm{He}\right)$. The cross-sections for NI scattering are taken from the work of Perkins and Cullen [7].

The third term in the left-hand side of Eq. (1) represents the diffusion in velocity space due to thermal conduction. To incorporate the unknown loss mechanism of energetic ions into the analysis, we simulate the velocitydependence of the energy-loss due to thermal conduction and the particle-loss time [19]. The source $\left(S_{i}(v)\right)$ and loss $\left(L_{i}(v)\right)$ terms take different form for every ion species $[5,17,19]$. By using computational iterative method, BFP equations for deuterons, tritons, alpha-particles, protons and ${ }^{3} \mathrm{He}$ are simultaneously solved for given electron temperature, fuel-ion densities and global confinement times, so that global power balance and particle conservation for each ion species are satisfied. Finally we obtain the velocity distribution functions at equilibrium state.

\subsection{Evaluation of neutron emission spec- trum}

From the obtained fuel-ion distribution functions, the neutron emission spectrum is evaluated as

$$
\begin{aligned}
\frac{\mathrm{d} N_{\mathrm{n}}}{\mathrm{d} E}(E)= & \iiint f_{\mathrm{D}}\left(\left|\vec{v}_{\mathrm{D}}\right|\right) f_{\mathrm{T}(\mathrm{D})}\left(\left|\vec{v}_{\mathrm{T}(\mathrm{D})}\right|\right) \\
& \times \frac{\mathrm{d} \sigma}{\mathrm{d} \Omega} \delta\left(E-E_{\mathrm{n}}\right) v_{\mathrm{r}} d \vec{v}_{\mathrm{D}} \mathrm{d} \vec{v}_{\mathrm{T}(\mathrm{D})} \mathrm{d} \Omega,
\end{aligned}
$$

where $N_{\mathrm{n}}$ represents the neutron generation rate, $\mathrm{d} \sigma / \mathrm{d} \Omega$ is the differential cross section of the $\mathrm{T}(\mathrm{d}, \mathrm{n})^{4} \mathrm{He}$ or $\mathrm{D}(\mathrm{d}, \mathrm{n})^{3} \mathrm{He}$ reaction and $E_{\mathrm{n}}$ represents the neutron energy in the laboratory system; the expression of which has been derived [20] as

$$
\begin{aligned}
E_{\mathrm{n}}= & \frac{1}{2} m_{\mathrm{n}} V_{\mathrm{c}}^{2}+\frac{m_{\alpha\left({ }^{3} \mathrm{He}\right)}}{m_{\alpha\left({ }^{3} \mathrm{He}\right)}+m_{\mathrm{n}}}\left(Q+E_{\mathrm{r}}\right) \\
& +V_{\mathrm{c}} \cos \theta_{\mathrm{c}} \sqrt{\frac{2 m_{\alpha\left({ }^{3} \mathrm{He}\right)} m_{\mathrm{n}}}{m_{\alpha\left({ }^{3} \mathrm{He}\right)}+m_{\mathrm{n}}}\left(Q+E_{\mathrm{r}}\right)},
\end{aligned}
$$

where $m_{\alpha(\mathrm{n})\left({ }^{3} \mathrm{He}\right)}$ is the $\alpha$-particle (neutron) $\left({ }^{3} \mathrm{He}\right)$ mass, $V_{\mathrm{c}}$ is the center-of-mass velocity of the colliding particles, $\theta_{\mathrm{c}}$ is the angle between $\vec{V}_{\mathrm{c}}$ and the $\alpha$-particle (neutron) velocity in the center-of-mass frame, $Q$ is the reaction $Q$ value, and $E_{\mathrm{r}}$ represents the relative energy of fuel-ions $E_{\mathrm{r}}=(1 / 2) m_{\mathrm{D}} m_{\mathrm{T}(\mathrm{D})} /\left(m_{\mathrm{D}}+m_{\mathrm{T}(\mathrm{D})}\right) \times\left|\vec{v}_{\mathrm{D}}-\vec{v}_{\mathrm{T}(\mathrm{D})}\right|^{2}$. Throughout the calculations, the cross sections for the $\mathrm{T}(\mathrm{d}, \mathrm{n})^{4} \mathrm{He}$ and $\mathrm{D}(\mathrm{d}, \mathrm{n})^{3} \mathrm{He}$ reactions are taken from the work of Drosg [21] and Bosch [22].

\section{Results and Discussion \\ 3.1 Distribution functions and neutron spec- trum}

Ion distribution functions for (a) deuterons, (b) triton, (c) ${ }^{3} \mathrm{He}$, (d) alpha-particles and (e) protons are shown in Fig. 1, when ${ }^{3} \mathrm{He}$ beam is injected into deuterium plasmas together with the ones when no external ${ }^{3} \mathrm{He}$-beam injection is made. In the calculation, the fuel-ion density $n_{\mathrm{D}}=8 \times 10^{19} \mathrm{~m}^{-3}$, energy and particle confinement times $\tau_{\mathrm{E}}=(1 / 2) \tau_{\mathrm{p}}=1 \mathrm{sec}$, beam-injection power $P_{\mathrm{NBI}}=2.5$ MW in a $100 \mathrm{~m}^{3}$ volume plasma are assumed. The electrons are assumed to be Maxwellian with $20 \mathrm{keV}$ temperature. We can find that the energetic non-Maxwellian component is created in proton distribution function due to its 2.45 and $14.67 \mathrm{MeV}$ fusion sources. Similarly to the knock-on tail formation by alpha-particles in DT plasmas [3-5], as a result of NI scattering of energetic protons by bulk deuterons, knock-on tails are created in deuteron velocity distribution function above $\sim 200 \mathrm{keV}$ energy range. The energetic component in proton distribution function above $2.5 \mathrm{MeV}$ energy range glows large as the ${ }^{3} \mathrm{He}$ beaminjection energy increases. Since the magnitude of the second knock-on tails (above $\sim 2 \mathrm{MeV}$ energy range) in deuteron distribution function are almost proportional to the magnitude of the second tail in proton distribution function (in $3 \sim 15 \mathrm{MeV}$ energy range), we can say that the second knock-on tail (above $\sim 2 \mathrm{MeV}$ energy range) in the deuteron distribution function is mainly caused by the external ${ }^{3} \mathrm{He}$-beam injection.

The neutron emission spectra by $\mathrm{D}(\mathrm{d}, \mathrm{n})^{3} \mathrm{He}$ reactions are shown in Fig. 2 (a) (corresponding to the calculations 

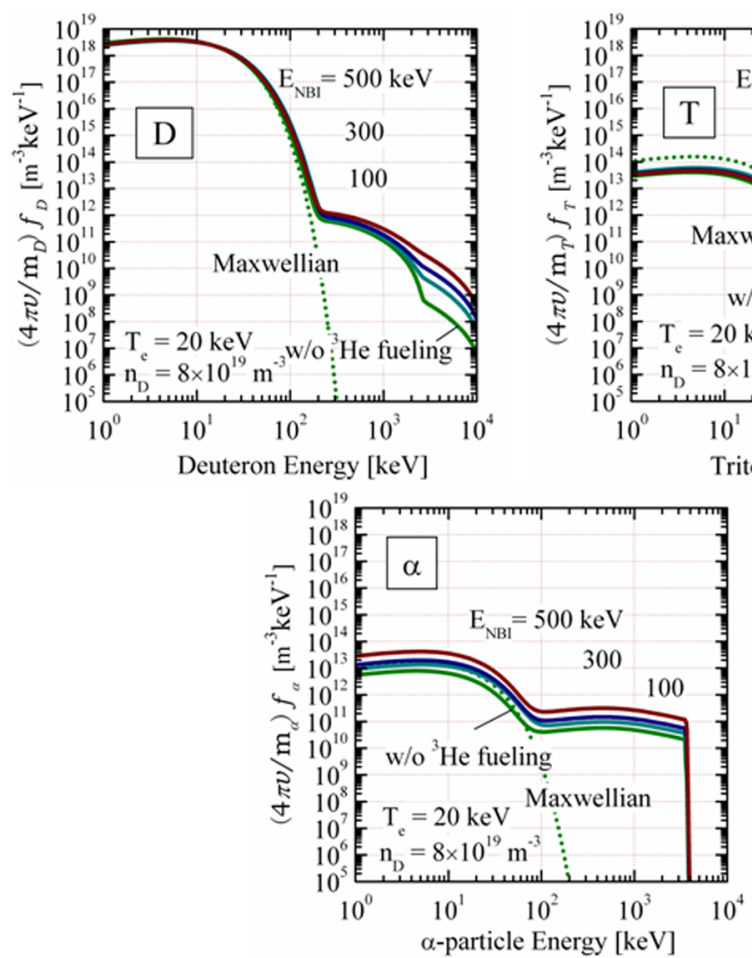
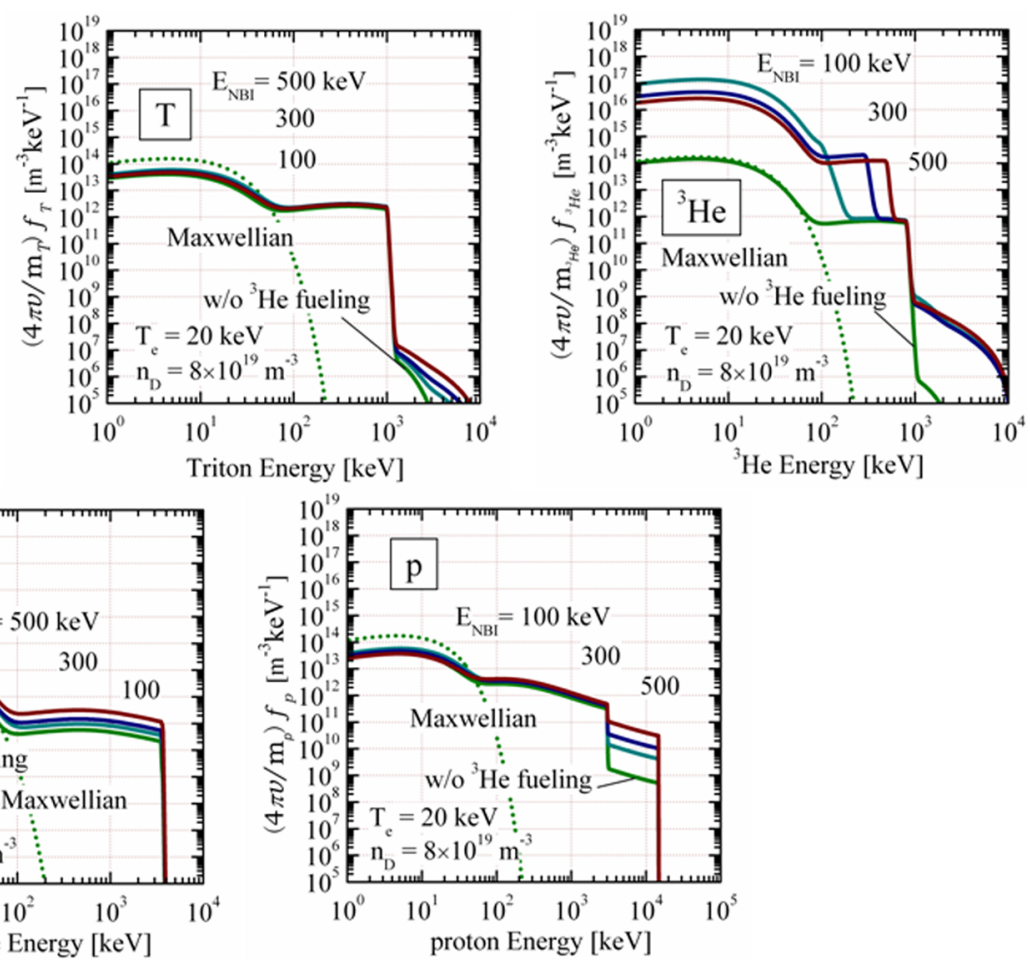

Fig. 1 Distribution functions of (a) deuterons, (b) tritons, (c) ${ }^{3} \mathrm{He}$, (d) alpha-particles and (e) protons in a typical deuterium plasma with ${ }^{3} \mathrm{He}$-beam injection. The electron temperature is $20 \mathrm{keV}$, deuteron density $n_{\mathrm{D}}=8 \times 10^{19} \mathrm{~m}^{-3}$, energy and particle confinement times $\tau_{\mathrm{E}}=(1 / 2) \tau_{\mathrm{p}}=1 \mathrm{sec}$ and beam-injection power $P_{\mathrm{NBI}}=2.5 \mathrm{MW}$ are assumed.

shown in Fig. 1). The dotted lines denote Gaussian distribution. It is found that the non-Gaussian component is formed in the emission spectrum and the recognizable increment in the neutron emission rate appears above $\sim 3 \mathrm{MeV}$ energy range. Since the increment of the neutron generation rate above $\sim 3 \mathrm{MeV}$ energy range is almost proportional to the ${ }^{3} \mathrm{He}$-beam injection energy, we can understand that the increment is caused by the knock-on tail formation in deuteron distribution function. The distinguishable change of the magnitude of the non-Gaussian component shown in Fig. 2 (a) would be suitable for the analysis of the transferred energy via NI scattering. In Fig. 2 (b) the neutron spectrum by $\mathrm{T}(\mathrm{d}, \mathrm{n})^{4} \mathrm{He}$ reactions are presented. We can observe the strongly broadened spectrum configuration. This is caused by the triton distribution function which has a large non-Maxwellian component produced by $1.01-\mathrm{MeV}$ fusion source (see Fig. 1). Since the degree of the distortion from Maxwellian in the triton distribution function is much greater than that in the deuteron distribution function, the effect of the knock-on tail formation in deuteron distribution function on the neutron spectrum by $\mathrm{T}(\mathrm{d}, \mathrm{n})^{4} \mathrm{He}$ does not significantly appear in Fig. 2(b). By comparing Fig. 2 (a) with Fig. 2 (b), we can see that the non-Gaussian component in $\mathrm{D}(\mathrm{d}, \mathrm{n})^{3} \mathrm{He}$ neutron spectrum is almost two orders smaller than that of $\mathrm{T}(\mathrm{d}, \mathrm{n})^{4} \mathrm{He}$ neutron spectrum. In Fig. 3 we also show the spectrum modification for several beam injection powers.
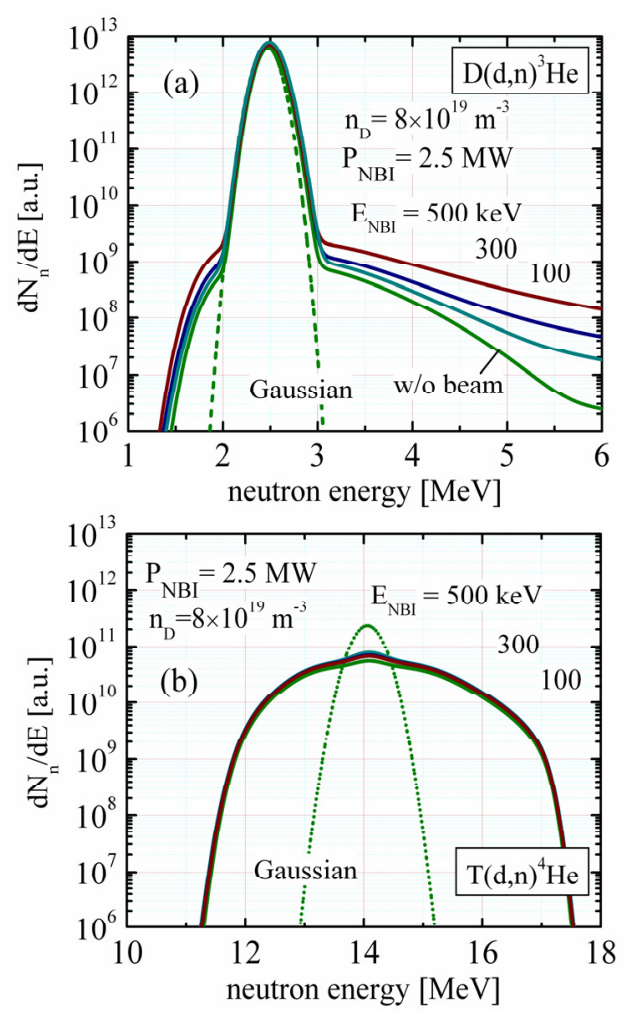

Fig. 2 Neutron emission spectra formed by (a) $\mathrm{D}(\mathrm{d}, \mathrm{n})^{3} \mathrm{He}$ and (b) $\mathrm{T}(\mathrm{d}, \mathrm{n})^{4} \mathrm{He}$ source neutrons when ${ }^{3} \mathrm{He}$ beam is injected into deuterium plasmas. 


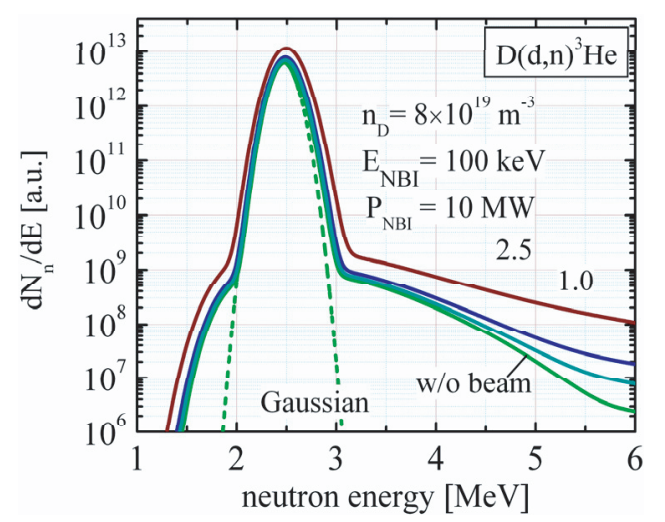

Fig. 3 Neutron emission spectrum formed by $D(d, n)^{3} \mathrm{He}$ reactions for several beam-injection powers.

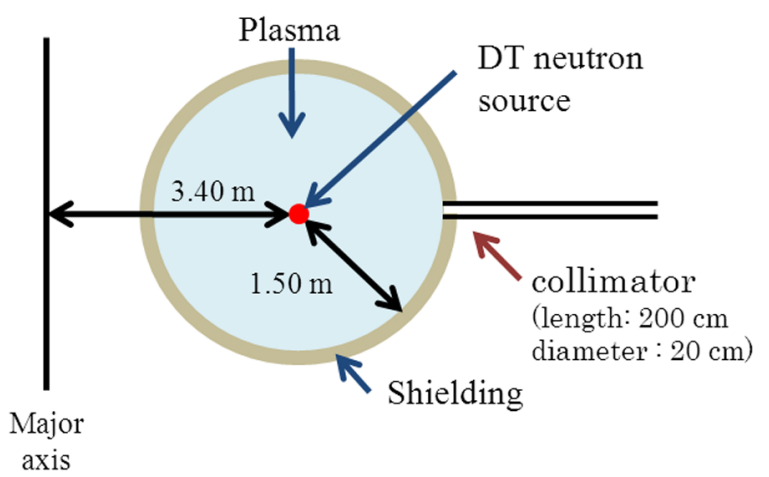

Fig. 4 Schematic view of the simplified model of the torus device assumed to evaluate the neutron flux at the neutron detector with collimator.

\subsection{Evaluation of noise neutrons for the measurement}

On the basis of the continuous-energy Monte Carlo transport code MVP [23], neutron flux in a typical torus device is estimated. In Fig. 4, the target device configuration assuming in our calculation is presented. We consider 3-dimentonal toroidal coordinate and source neutrons are assumed to be generated on the minor axis with Gaussian distribution at almost the same generation rate shown in Fig. 2. The size and structural materials of device and shielding are chosen referring to the design parameter of JT60SA [24] (Vacuum vessel: $1500 \mathrm{~mm}$ radius, Shielding: $25 \mathrm{~mm}$ first wall, $39 \mathrm{~mm}$ SUS316, $140 \mathrm{~mm}$ boron water and $24 \mathrm{~mm}$ SUS316 layer from the plasma). The neutron flux when we chose graphite or SUS316 as a structural material of the $25 \mathrm{~mm}$ first wall are shown in Fig. 5 together with the neutron flux to be observed at the detector with neutron collimator (red circles), i.e. length $200 \mathrm{~cm}$ and diameter $20 \mathrm{~cm}$. A few peaks can be seen in the neutron flux when graphite first wall is assumed. This is caused by the neutron generation reactions of graphite. We can see that the relative intensity of the neutron flux at $3 \sim 6 \mathrm{MeV}$ energy range is reduced almost two orders from the value at the $14-\mathrm{MeV}$ source energy range. When we use a collima-

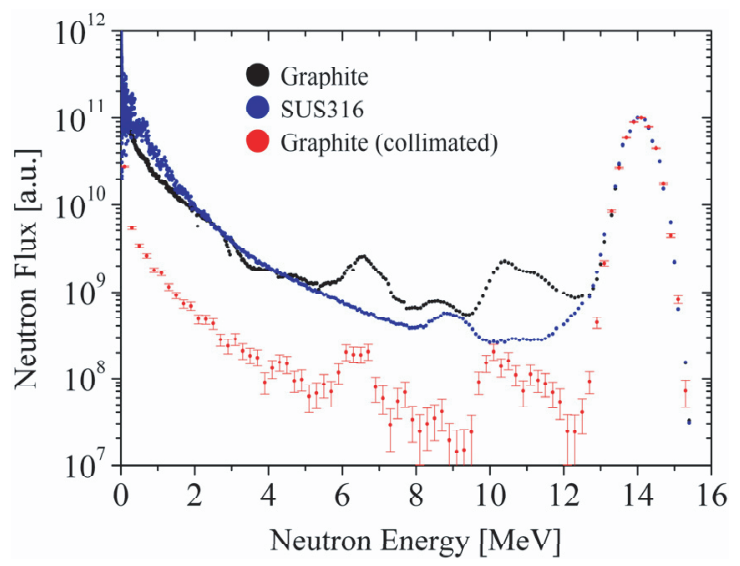

Fig. 5 Relative intensity of source and slowing-down neutron flux arising from $\mathrm{T}(\mathrm{d}, \mathrm{n})^{4} \mathrm{He}$ reactions. The red circles indicate the neutron flux to be measured at the detector with collimator.

tor to remove the noise neutrons not directly arriving from the plasma, we can further reduce the noise component. As shown in Fig. 5 the noise neutrons in $3 \sim 6 \mathrm{MeV}$ energy range can be reduced more than three orders from the values at the $14-\mathrm{MeV}$ neutron source energy, and we can distinguish the non-Gaussian component shown in Figs. 2 and 3 from the noise neutrons arising from $\mathrm{T}(\mathrm{d}, \mathrm{n})^{4} \mathrm{He}$ reactions.

\section{Conclusion}

A possible experiment to validate the NI-scattering effects in $\mathrm{D}^{3} \mathrm{He}$ plasmas was proposed. As a result of knockon tail formation in deuteron distribution function due to NI scattering with energetic protons, non-Gaussian component in neutron spectrum formed by $\mathrm{D}(\mathrm{d}, \mathrm{n})^{3} \mathrm{He}$ reactions appears. We tried to increase the magnitude of the non-Gaussian component by using ${ }^{3} \mathrm{He}$-beam injection and tried to reduce the noise neutrons arising from $\mathrm{T}(\mathrm{d}, \mathrm{n})^{4} \mathrm{He}$ reactions by the neutron collimator. In the previous paper [18] we also proposed a measuring method using $\gamma$-ray generating ${ }^{6} \mathrm{Li}+\mathrm{D}$ reactions. By using the $\gamma$-ray generating reactions in parallel with the neutron spectrum observation, more precise analysis could be possible. The data on some conditions of interest may exist in the past experiments made on existence fusion devices, e.g., JET. The detailed search of the stored data may also be useful for the validations.

[1] Y. Nakao et al., Fusion Technol. 27, 555 (1995).

[2] H. Matsuura et al., Proc. ICENES'93 (Chiba, 1993), edited by H.Yasuda (World Scientific, Singapore, 1994) p.266.

[3] L. Ballabio et al., Phys. Rev. E 55, 3358 (1997).

[4] J. Källene et al., Phys. Rev. Let. 85, 1246 (2000).

[5] H. Matsuura et al., Phys. Plasmas 13, 62507 (2006).

[6] J.J. Devaney et al., Nucl. Sci. Eng. 46, 323 (1971).

[7] S.T. Perkins et al., Nucl. Sci. Eng. 20, 77 (1981).

[8] Y. Nakao et al., Nucl. Fusion 21, 979 (1981).

[9] J. Galambos et al., Nucl. Fusion 24, 739 (1984). 
[10] Y. Nakao et al., Nucl. Fusion 28, 1029 (1988).

[11] H. Matsuura et al., Plasma Fusion Res. 7, 2403076 (2012).

[12] D. Ryutov, Phys. Scr. 45, 153 (1992).

[13] M. Helander et al., Plasma Phys. Control. Fusion 35, 367 (1993).

[14] R.K. Fishier et al., Nucl. Fusion 34, 1291 (1994).

[15] A.J.H. Donné et al., Nucl. Fusion 47, S337 (2007).

[16] H. Matsuura et al., Phys. Plasmas 16, 0542507 (2009).

[17] H. Matsuura et al., Plasma Phys. Control. Fusion 53,
035023 (2011).

[18] H. Matsuura et al., Fusion Sci. Technol. 60, 634 (2011).

[19] E. Bittoni et al., Nucl. Fusion 29, 931 (1980).

[20] H. Brysk, Plasma Phys. 15, 611 (1973).

[21] M. Drosg and O. Schwerer, Handbook of Nuclear Activation Data, IAEA, Vienna, 1, 111 (1987).

[22] H.-S. Bosch et al., Nucl. Fusion 32, 611 (1992).

[23] Y. Nagaya et al., JAERI-1348 (2005).

[24] A.M. Sukegawa et al., Fusion Eng. Des. 82, 2799 (2007). 